\title{
Fibre-optic delivery of time and frequency to VLBI station
}

\author{
P. Krehlik ${ }^{1}$, Ł. Buczek ${ }^{1}$, J. Kołodziej ${ }^{1}$, M. Lipiński ${ }^{1}$, Ł. Śliwczyński ${ }^{1}$, J. Nawrocki $^{2}$, P. Nogaś ${ }^{2}$, A. Marecki ${ }^{3}$, \\ E. Pazderski ${ }^{3}$, P. Ablewski ${ }^{4}$, M. Bober ${ }^{4}$, R. Ciuryło ${ }^{4}$, A. Cygan ${ }^{4}$, D. Lisak ${ }^{4}$, P. Masłowski ${ }^{4}$, P. Morzyński $^{4}$, \\ M. Zawada ${ }^{4}$, R. M. Campbell ${ }^{5}$, J. Pieczerak ${ }^{6}$, A. Binczewski ${ }^{7}$, and K. Turza ${ }^{7}$ \\ 1 AGH University of Science and Technology, Mickiewicza 30, 30-059 Kraków, Poland \\ e-mail: krehlik@agh.edu.pl \\ 2 Astrogeodynamic Observatory (AOS), Borowiec, Drapałka 4, 62-035 Borowiec, Poland \\ 3 Centre for Astronomy, Faculty of Physics, Astronomy and Informatics, Nicolaus Copernicus University, Grudziądzka 5, \\ 87-100 Toruń, Poland \\ e-mail: amr@astro.uni.torun.pl \\ 4 Institute of Physics, Faculty of Physics, Astronomy and Informatics, Nicolaus Copernicus University, Grudziądzka 5, \\ 87-100 Toruń, Poland \\ 5 Joint Institute for VLBI ERIC, Postbus 2, 7990 AA Dwingeloo, The Netherlands \\ 6 Orange Polska S.A., Obrzeżna 7, 02-691 Warsaw, Poland \\ 7 Poznań Supercomputing and Networking Center, Jana Pawła II 10, 61-139 Poznań, Poland
}

Received 14 February 2017 / Accepted 17 March 2017

\begin{abstract}
The quality of Very Long Baseline Interferometry (VLBI) radio observations predominantly relies on precise and ultra-stable time and frequency (T\&F) standards, usually hydrogen masers (HM), maintained locally at each VLBI station. Here, we present an operational solution in which the VLBI observations are routinely carried out without use of a local HM, but using remote synchronization via a stabilized, long-distance fibre-optic link. The T\&F reference signals, traceable to international atomic timescale (TAI), are delivered to the VLBI station from a dedicated timekeeping laboratory. Moreover, we describe a proof-of-concept experiment where the VLBI station is synchronized to a remote strontium optical lattice clock during the observation.
\end{abstract}

Key words. instrumentation: high angular resolution - instrumentation: interferometers

\section{Introduction}

There is one crucial technical difference between Very Long Baseline Interferometry (VLBI) and the interferometric techniques used in radio astronomy in case of small (tens of kilometres) or medium-sized (hundreds of kilometres) baselines. This difference is that in VLBI the electromagnetic signals received by the individual radio telescopes are not correlated during the observation itself but "off-line", that is, they are recorded, then the media are shipped to and played back at the correlator to finally acquire the values of fringe visibility function. To preserve the mutual coherence of the signals recorded at all stations, the receivers and the data formatters have to be synchronized to ultra-stable time and frequency (T\&F) standards. Each station has its own T\&F standard. The common practice is to use hydrogen masers (HM) because they are affordable and their shortterm stability appears sufficient for this purpose.

With the advent of very fast fibre-optic links, the above paradigm of VLBI has changed dramatically. In brief, owing to the availability of high-speed links, the VLBI resembles the short-base ("local") radio interferometry more than ever before. The breakthrough is implementation of the so-called e-VLBI, where the data correlation is performed in real time, meaning without recording and shipping of the media. But even in e-VLBI, each of the participating stations still relies on its own T\&F standard to which it is synchronized. This, however, may change too. New opportunities arose because of the development of novel techniques of high-precision fibre-optic distribution of $\mathrm{T} \& \mathrm{~F}$ signals over distances of hundreds of kilometres. The advantages of this approach can be summarized as follows: (1) stations may be synchronized by remote $T \& F$ standards so they no longer need to install and maintain their own; (2) many stations may receive synchronization signal traceable to a common international atomic timescale (TAI); and (3) some stations may get access to new generation optical atomic clocks, outperforming traditional HMs in terms of both stability and absolute accuracy. As optical atomic clocks are rapidly becoming the most stable clocks and have already significantly surpassed their microwave counterparts (Hinkley et al. 2013; Le Targat et al. 2013; Bloom et al. 2014; Falke et al. 2014; Ushijima et al. 2015), the last point emerges as particularly applicable to an actively developed millimetre-wavelength VLBI, already limited by the stability of HMs for required integration times (Rioja et al. 2012; Rogers et al. 1981; Thompson et al. 1986).

Here, we present the basics of fibre-optic systems dedicated for distribution of T\&F reference signals (Sect. 2). Next, we describe the solution implemented in Poland where a fully operational delivery of UTC-traceable T\&F signals from the Time Laboratory of the Astrogeodynamical Observatory in Borowiec near Poznan (AOS) to the VLBI station in Piwnice near Toruń operated by the Centre for Astronomy of Nicolaus Copernicus University in Toruń (hereafter: Toruń VLBI station) has been established over a 345-km-long fibre link (Sect. 3). In Sect. 4, we present a proof-of-concept experiment where the Toruń VLBI 


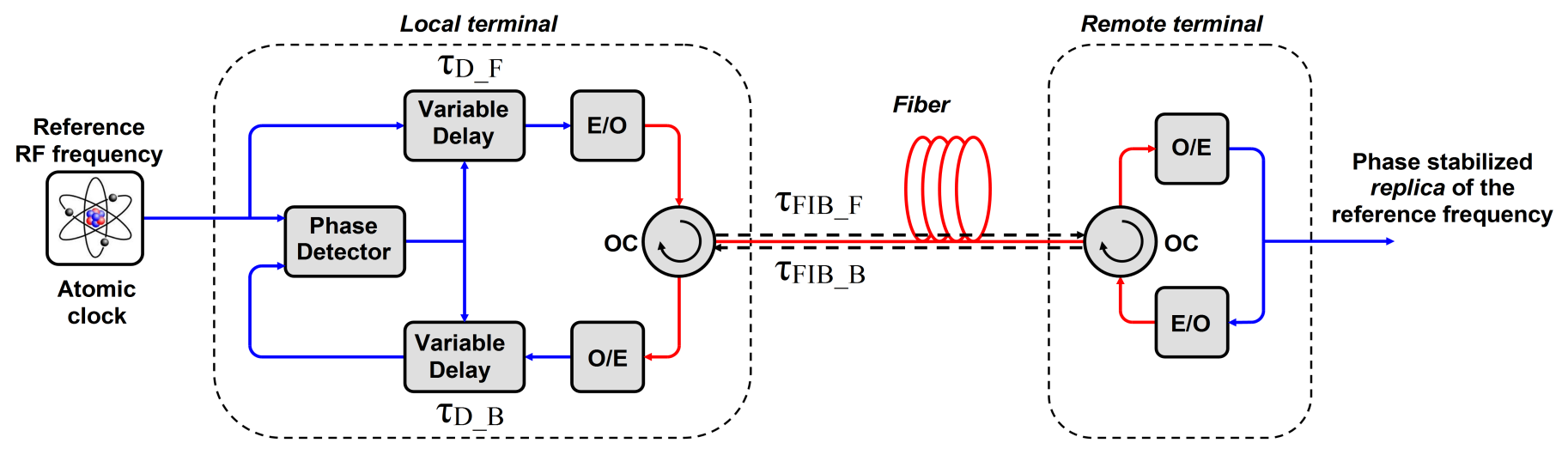

Fig. 1. Simplified block diagram of the ELSTAB system; E/O stands for an intensity-modulated laser, O/E - an optical-to-electrical receiver, OC an optical circulator.
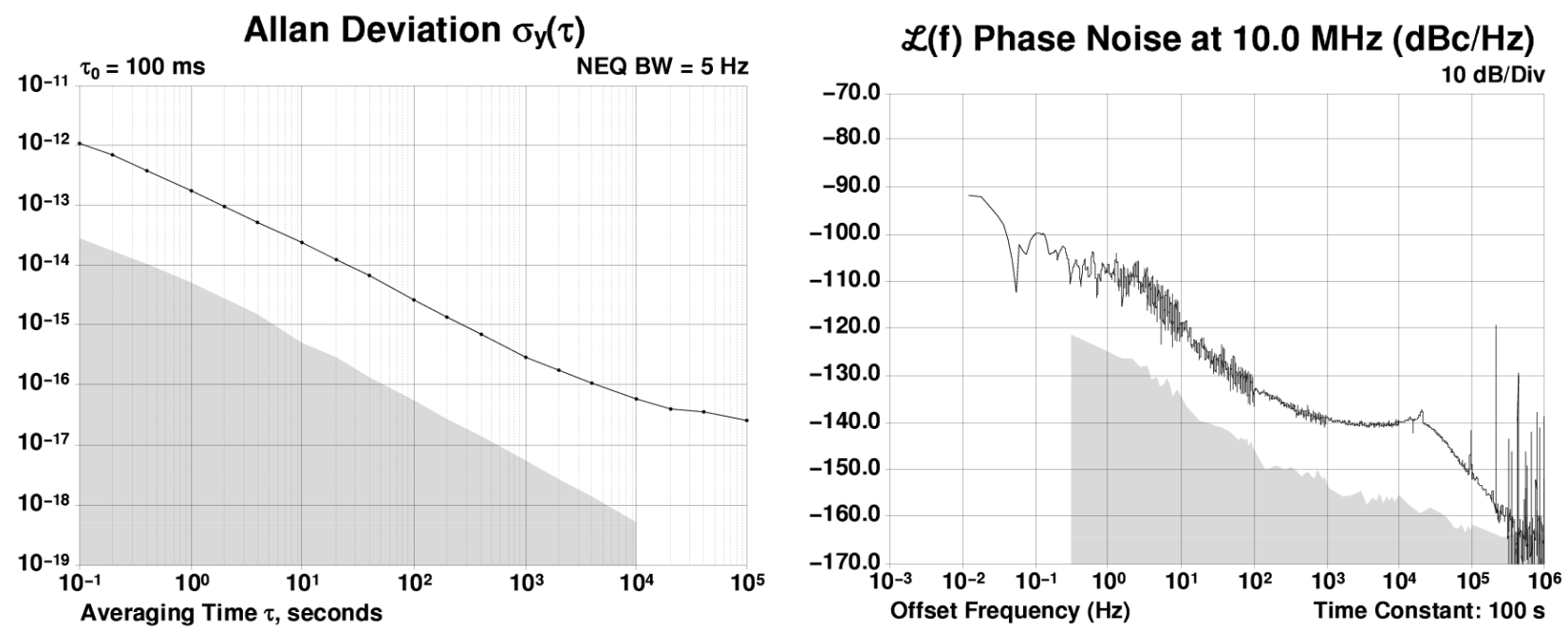

Fig. 2. Allan deviation (left) and phase noise (right) introduced by ELSTAB.

station was synchronized with a remote strontium optical lattice clock developed at National Laboratory for Atomic, Molecular and Optical Physics (KL FAMO). Our results are summarized in Sect. 5.

\section{Fibre optic distribution of frequency and time}

During the last decade, optical fibre has been recognized as a very powerful medium for long-distance distribution of the highest quality T\&F signals, generated in metrological institutions dedicated to developing and maintaining atomic clocks and generating atomic timescales. Many such links, at both experimental and operational levels, have been recently established (Lopez et al. 2010, 2012; Droste et al. 2013; Śliwczyński et al. 2013; Calonico et al. 2014; Dierikx et al. 2016). There are, however, specific issues related to vulnerability of the fibres to environmental factors that should be addressed to ensure high stability of delivered synchronization signals. The most pronounced effect is the thermal instability of the propagation delay that affects both phase and frequency of the carrier signal and also the temporal positions of received time-stamps. The reason for this effect is mainly the thermal dependence of the refractive index of silica glass and - to a smaller extent - variation of the physical length of the fibre. For various types of telecommunicationgrade fibres the related coefficient of the delay fluctuations is close to $40 \mathrm{ps} \mathrm{K}^{-1} \mathrm{~km}^{-1}$ (Śliwczyński et al. 2010). Other important environmental factors are variable tensions and vibration affecting the cable that also modulate the propagation delay. The power spectral density of this "acoustic noise" is usually located in the range between 10 and $40 \mathrm{~Hz}$ and is strongly dependent on the fibre location: the noise is much more pronounced in urban areas and especially in fibres deposited along railways. A very specific case is also aerial-suspended fibres, directly exposed to diurnal temperature fluctuations and solar heating, winds, and vibrations generated by high-voltage power lines (Śliwczyński et al. 2016).

The common method used to overcome these environmental limitations is to arrange a feedback system in which the signal is sent to the remote (user) side, where it is redirected backward to cancel the delay fluctuations in a closed loop topology. The cancellation of the delay fluctuations may be performed directly in the optical or electrical domain (Ma et al. 1994; Lopez et al. 2010; Droste et al. 2013; Śliwczyński et al. 2013), or realized at the software (protocol) level (Dierikx et al. 2016).

In our solution called ELSTAB (see Fig. 1), the fluctuations of propagation delay are compensated electronically by means of the two precisely matched variable delay lines and the phase detector that controls the delay lines to keep the round trip phase relation (and thus also the round trip delay) constant, unaffected by the fluctuations of the fibre delay. This may be written as:

$\tau_{\mathrm{D} \_\mathrm{F}}+\tau_{\mathrm{FIB} \_\mathrm{F}}+\tau_{\mathrm{D} \_\mathrm{B}}+\tau_{\mathrm{FIB} \_\mathrm{B}}=$ const.

where $\tau_{D_{-} F}$ and $\tau_{D_{-} B}$ are (variable) delays of the delay blocks and $\tau_{\text {FIB_F}}, \tau_{\text {FIB_B }}$ are the propagation delays of the fibre for the 


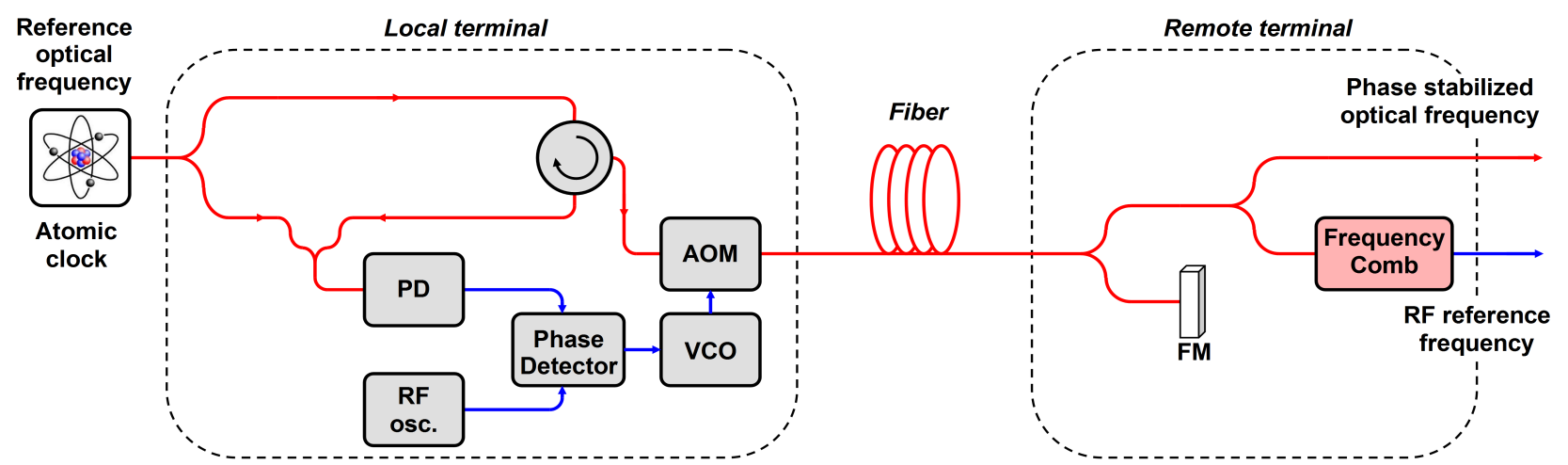

Fig. 3. Simplified block diagram of the stabilized distribution of the optical carrier frequency; AOM stands for an acousto-optic modulator, VCO a voltage controlled oscillator, PD - a photo-diode, and FM - a Faraday mirror.

forward and backward directions, respectively. Assuming that the fibre delay variations are the same in both directions

$\Delta \tau_{\text {FIB_F }}=\Delta \tau_{\text {FIB_B }}$

and that the tuning characteristic of both delay blocks are identical

$\Delta \tau_{\mathrm{D} \_\mathrm{F}}=\Delta \tau_{\mathrm{D} \_\mathrm{B}}$,

it may also be noticed that the one-way delay from the input to the remote output, determined by the sum: $\tau_{D_{F} F_{1}}+\tau_{\text {FIB F }}$, is constant. (For more detailed analysis and discussion, see Śliwczyński et al. 2011.) The typical stability of the $10 \mathrm{MHz}$ frequency transfer is shown in Fig. 2. The system-induced Allan deviation is about $2-4 \times 10^{-13}$ for one-second averaging and goes down with a slope close to $\tau^{-1}$ where $\tau$ is an observation period. The introduced phase noise is about $-105 \mathrm{dBc} / \mathrm{Hz}$ for a $1 \mathrm{~Hz}$ offset and about $-140 \mathrm{dBc} / \mathrm{Hz}$ for a $1 \mathrm{kHz}$ offset. Additionally, the full ELSTAB system also comprises the time distribution facility, achieved by embedding the time-stamps (1 PPS signal), whose offset (delay) with respect to the UTC reference may be calibrated with an uncertainty well below 100 ps (Krehlik et al. 2012). In the case of long-haul links, dedicated bidirectional optical amplifiers should be installed to compensate the fibre attenuation (Śliwczyński et al. 2013).

A fundamentally different approach for frequency distribution is usually used when an optical clock is the source of reference signal. In this case, the optical frequency generated in the clock system is directly launched into the fibre and basically no modulation is applied. As the environmental factors that influence the fibre affect the optical carrier frequency observed at the output, such a link should also be stabilized. The typical solution is presented in Fig. 3. The optical carrier is transmitted to the remote terminal, where some part of the signal is redirected backward using a Faraday mirror. The frequency of the light coming back to the local terminal is compared with the reference provided directly from the clock, thus the frequency fluctuations induced in the fibre may be detected and corrected. The corrections are performed with an acousto-optic modulator that shifts the phase/frequency of the light going both in forward and backward directions (Droste et al. 2013; Calonico et al. 2014). Such systems allow full exploitation of the superior stability of the optical clocks, but it should be borne in mind that the "natural" output of the system is the $190 \mathrm{THz}$ optical frequency that cannot directly be used in the electrical domain. It may be converted to electrical RF domain by an optical frequency comb (Newbury \& Swann 2007), this, however, seriously increases the cost and complexity of the overall installation. Nevertheless, first experiments using this technique for characterization of a $\mathrm{HM}$ at the Medicina VLBI station (Clivati et al. 2015) and to synchronize a VLBI session (Perini et al. 2016; Clivati et al. 2017) have been performed.

From a practical point of view, the main concern is the compatibility of the frequency and time distribution with other services carried out in the fibre-optic network. The simplest solution, applicable when spare fibres are available in an optical cable, is to use such a fibre solely for frequency and time distribution - see Fig. 4a. In the case of a long-distance link (longer than approximately $100 \mathrm{~km}$ ), bidirectional optical amplifiers should be installed at spacings of $40-70 \mathrm{~km}$. This solution, although not effective in terms of fibre usage, is preferred by network operators as it does not interfere with standard telecommunications services. Sharing the fibre used for other services is also possible by means of wavelength division multiplexing (WDM), commonly used in optical networks. Typically, the wavelength grid is organized with $100 \mathrm{GHz}$ or $50 \mathrm{GHz}$ channel spacing and one or two channels needed for frequency and time distribution may be selected by optical filters (add-drop multiplexers) and processed (amplified) independently to other wavelengths dedicated for standard services - see Fig. 4b. It should be stressed, however, that in long-haul optical networks fibres are used in pairs, one fibre for each direction, whereas in our case the preferable option is to use the same fibre for both directions. Even though the solution depicted in Fig. $4 \mathrm{~b}$ was demonstrated to be safe for telecommunication traffic and to maintain frequency and time distribution stability (Lopez et al. 2012), it is still hardly accepted within the community of network operators. The last option in the case of lack of spare fibres is to use so-called alienlambda scenario where the synchronization signals are transmitted on dedicated wavelength in two parallel fibres, and routed and amplified by a standard telecommunication equipment located in the network nodes, similarly as other services - see Fig. 4c. A serious drawback of this solution is degradation of the forward-backward propagation symmetry that was the underlying assumption of the stabilized frequency and time distribution; the behaviour of parallel fibres located in the same cable can still be correlated, but distinct network equipment in the nodes for each direction causes severe stability degradation (Śliwczyński et al. 2015).

\section{UTC traceable, remote synchronization of the Torun VLBI station}

In 2015, the Polish fibre-optic network for T\&F distribution (OPTIME) reached Nicolaus Copernicus University in Torun (Buczek et al. 2015) and the Toruń VLBI station was connected 


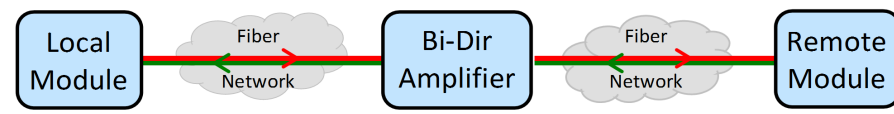

a)

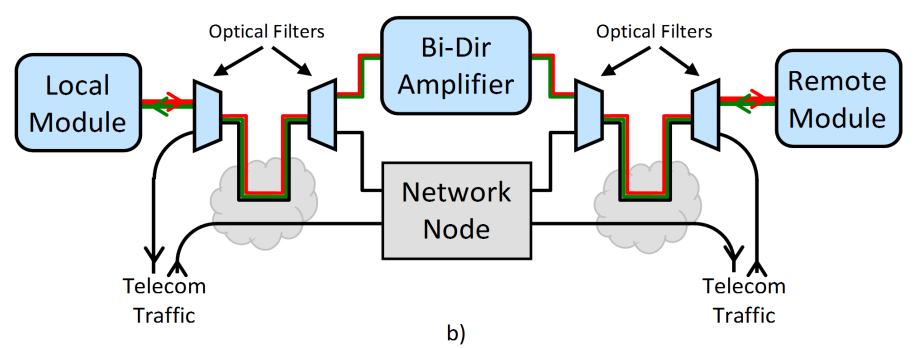

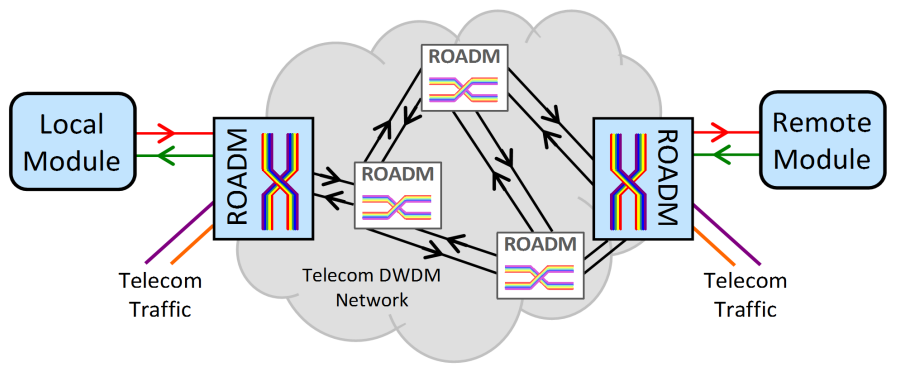

c)

Fig. 4. Possible strategies for frequency and time distribution exploiting fibre optic telecommunication infrastructure: a) dark-fibre approach, b) dark-channel approach and c) alien-lambda approach. ROADM stands for re-configurable optical add-drop multiplexer.

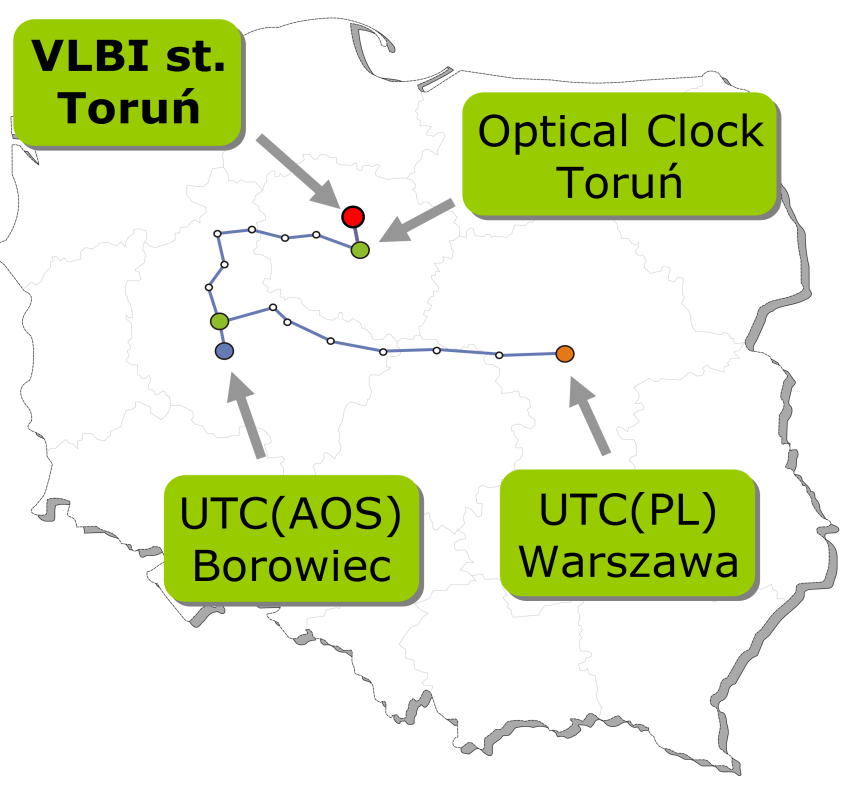

Fig. 5. VLBI station in Piwnice near Torun connected to the fibre optic T\&F distribution network developed under the OPTIME project.

to the backbone with a $15-\mathrm{km}$-long slave link. This created an opportunity to synchronize the Torun VLBI station to one of the Polish UTC laboratories, UTC (AOS) or UTC (PL), and alternatively to the strontium optical lattice clock developed at KLFAMO in Toruń (Bober et al. 2015) - see Fig. 5. The total length of the synchronizing path is approximately $350 \mathrm{~km}$. Physically, the T\&F signals are generated at AOS and are based on an HM working as a flywheel, but it should be stressed that the HM output is precisely corrected to obtain $\mathrm{T} \& \mathrm{~F}$ references UTC (AOS) - coherent with the TAI. Therefore, the signals received at the Torun VLBI station are both stable and UTCtraceable.

We conducted a dedicated VLBI experiment on 18 December 2015 to serve as a proof-of-concept to evaluate the quality of the remote synchronization and hence its suitability for operational use in VLBI experiments. Besides the Torun VLBI station, the participating telescopes included Westerbork (NL), Medicina (IT), and Yebes (ES). The observing format comprised four single-polarization $8 \mathrm{MHz}$ sub-bands, together spanning 4974.49 MHz to 5006.49 MHz. The experiment lasted for three hours, continually tracking the bright radio quasar $2007+777$. The raw data from all telescopes were transferred over optical fibre to the Joint Institute for VLBI ERIC (JIVE) and the correlation proceeded in real time, that is, using the e-VLBI technique.

During the experiment, the quality of the remote synchronization was evaluated by dividing the experiment into two domains: first when the T\&F signal came from the local HM of the Torun VLBI station and second when this was swapped out for the remote HM at AOS provided via fibre. We then compared the behaviour of the residual fringe phase in the individual baselines/sub-bands in each "domain". In addition to being correlated in the e-VLBI mode, about $45 \mathrm{~min}$ of the in-streaming data from the telescopes for each of the two domains were also recorded at JIVE to enable subsequent re-correlations using different coherent integration intervals. The correlator model (Gordon et al. 2006) included the standard geometric, relativistic, and propagation terms, plus a first-order polynomial clock model per telescope. The a priori rate coefficients of these clock models were set by linear fits to a few day's worth of monitoring the telescopes' maser outputs against GPS. At the beginning of each domain, we adjusted the clock offset and rate coefficients to flatten residual delays and rates by correlating a few minutes of data. We draw the reader's attention to this process here because the local HM at the Torun VLBI station had developed a large rate bias (about $-19.9 \mathrm{ps} / \mathrm{s}$ at the time of this experiment) that was removed by this adjustment procedure. The remote HM showed no significant residual rate with respect to UTC.

Because the two domains are intrinsically distinct time ranges, the phases on the baselines to Torun cannot simply be subtracted to compare directly the two methods of T\&F control at the Torun VLBI station: earth rotation will lead to a changing projection of a baseline with respect to the radio source structure on the plane of the sky and unmodelled propagation effects along the lines of sight from the individual telescopes will also evolve (the more important effect for the time-scales involved here). Figure 6 shows an example of the behaviour of the residual interferometer phase, plotting one 15 min scan from each domain, using the middle two sub-bands.

We considered two phase-difference quantities to characterize the phase noise achieved for each method of T\&F control at Torun, using both 2 - and $4 \mathrm{~s}$. integration intervals: first, a timeseries of the differences of consecutive phases, and second, a 
Phase for Local Maser

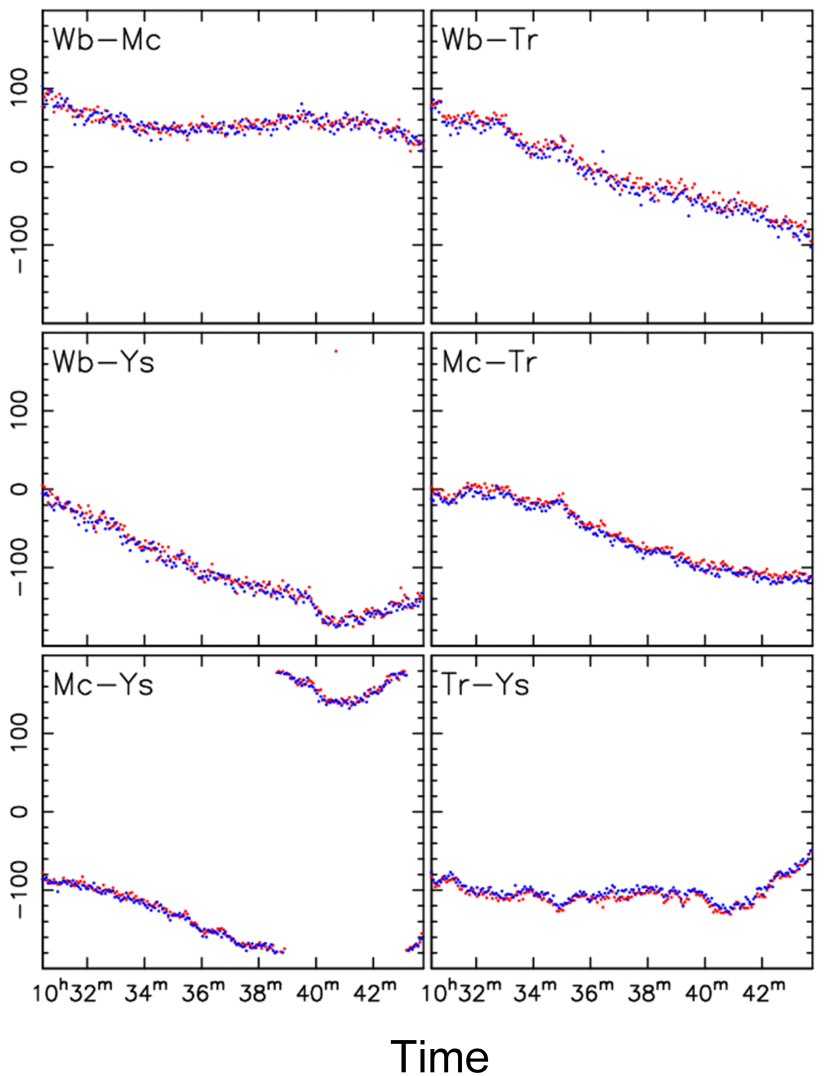

Phase for Remote Maser

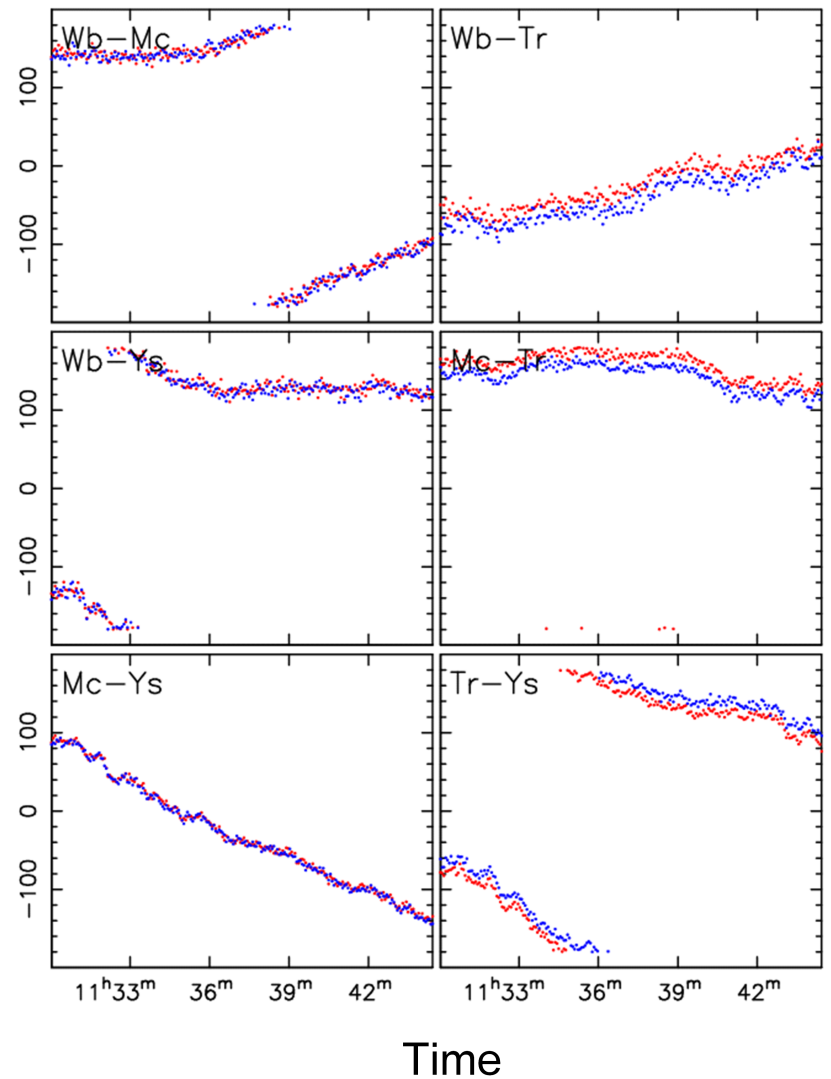

Fig. 6. Residual interferometer phase for two methods of providing T\&F control to the Torun VLBI station during the experiment of 18 December 2015. The $x$-axis is UTC and the $y$-axis is degrees of phase in the middle two sub-bands (colour-coded), vector averaged over the middle $80 \%$ of each sub-band. Each four-second integration interval is plotted as a separate point. The baselines are arranged such that those including Torun are in the right-hand column for each of the T\&F control methods.

time series of the difference between a phase and the linear interpolation between the phases of the two surrounding integration intervals. In each case, the time-series were split into two or three subsets, respectively, such that each individual integration contributed to only one difference pair or triplet within a given subset. We then compared the rms of each subset as a means to characterize the phase noise, independent of any remaining unmodelled phase slopes across the full time range considered. Table 1 shows the results for the case of the difference-pair statistic with the two subsets labelled as "odd" and "even" referring to the parity of the first point in each pair comprising the subset. The relative magnitude of these phase-noise statistics across the various baselines is in line with the sensitivities of the constituent telescopes. The very similar results for the two domains strongly suggests that the phase noise is dominated by factors unrelated to the specific method of T\&F control at the Torun VLBI station and that the remote HM at AOS can provide the operational synchronization for VLBI experiments.

Thus, after the successful completion of this experiment, it was decided to permanently switch the synchronization at the Torun VLBI station to the remote HM. The motivation for this was that the local HM that had provided T\&F control for the Torun VLBI station for 20 years had developed the aforementioned worsening drift and its maintenance had become troublesome, calling into question its operational reliability. In the end, all regular VLBI sessions carried out since the beginning of 2016 have been performed with the remote synchronization provided over the fibre from UTC(AOS) laboratory.

\section{Experimental synchronization with strontium optical lattice clock}

It has been recently shown that in millimetre and sub-millimetre VLBI the losses induced by HM instabilities are comparable to those from high-quality tropospheric conditions (Rioja et al. 2012) and significant improvement in sensitivity can be expected after replacing a traditional HM by a more stable frequency standard, for example, an optical clock. However, the low-noise down-conversion of the optical frequency to RF or microwave domain is essential for exploiting the superior stability of the optical clock.

In our proof-of-concept experiment we used an strontium optical lattice clock developed and operated at KLFAMO (Morzyński et al. 2015) to synchronize Toruń VLBI station during a test session as shown in Fig. 7. The station was referenced to the clock over an optical frequency comb system and a $15 \mathrm{~km}$ long ELSTAB-based fibre link. The optical frequency comb was locked to the $698 \mathrm{~nm}$ transition $(429228066418008.3(2.1) \mathrm{Hz})$ from the optical clock. Both repetition rate and offset frequency of the comb were stabilized to the beat-note signal of one of the comb tooth and the so-called clock laser. The clock laser used in our optical atomic clock is stabilized to the ultra-stable 

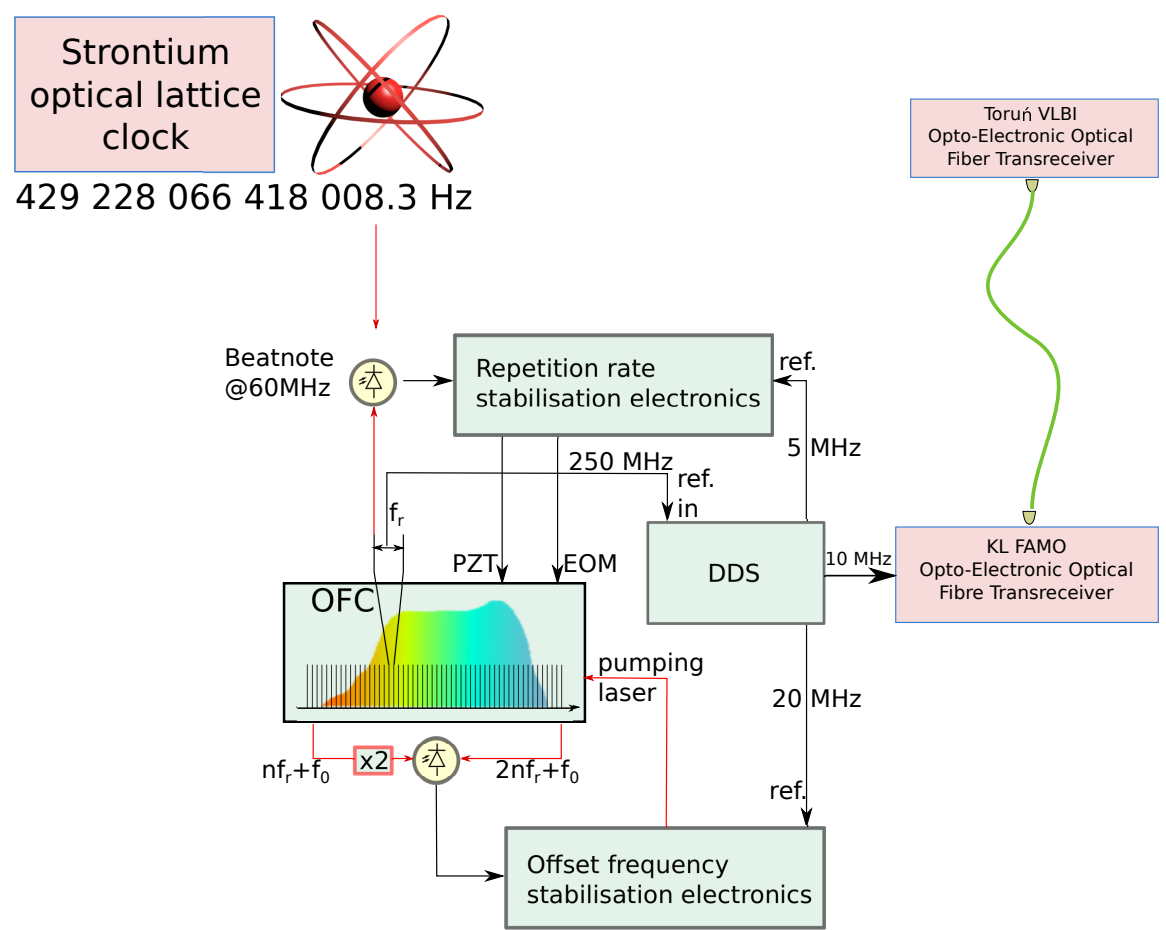

Fig. 7. Toruń VLBI station referenced to a strontium optical lattice clock over an optical frequency comb system and a $15 \mathrm{~km}$-long ELSTAB-based fibre link. $f_{\mathrm{r}}$ and $f_{\mathrm{o}}$ are the repetition and offset frequencies of the comb, respectively. OFC stands for an optical frequency comb and DDS stands for a direct digital synthesizer.

Table 1. Phase noise in picoseconds for different baselines and subbands for local and remote HM.

\begin{tabular}{|c|c|c|c|c|c|}
\hline \multirow[t]{4}{*}{ Baseline } & \multirow{4}{*}{$\begin{array}{l}\text { Sub- } \\
\text { band } \\
\text { and } \\
\text { polarity }\end{array}$} & \multicolumn{2}{|c|}{ Local HM } & \multicolumn{2}{|c|}{ Remote HM } \\
\hline & & noise & noise & noise & noise \\
\hline & & $\mathrm{rms}$ & $\mathrm{rms}$ & $\mathrm{rms}$ & $\mathrm{rms}$ \\
\hline & & odd & even & odd & even \\
\hline Wb-Tr & ORR & 4.928 & 4.331 & 5.045 & 4.949 \\
\hline $\mathrm{Wb}-\mathrm{Tr}$ & $1 \mathrm{RR}$ & 5.062 & 5.130 & 5.152 & 5.186 \\
\hline Wb-Tr & 2RR & 5.318 & 5.048 & 5.015 & 5.950 \\
\hline $\mathrm{Wb}-\mathrm{Tr}$ & $3 R R$ & 4.169 & 5.032 & 5.475 & 4.518 \\
\hline $\mathrm{Mc}-\mathrm{Tr}$ & ORR & 2.643 & 2.721 & 2.687 & 2.879 \\
\hline $\mathrm{Mc}-\mathrm{Tr}$ & $1 \mathrm{RR}$ & 2.929 & 2.605 & 3.151 & 3.230 \\
\hline $\mathrm{Mc}-\mathrm{Tr}$ & 2RR & 2.840 & 2.488 & 2.993 & 3.041 \\
\hline $\mathrm{Mc}-\mathrm{Tr}$ & $3 \mathrm{RR}$ & 3.004 & 2.855 & 2.764 & 3.218 \\
\hline Tr-Ys & ORR & 2.799 & 2.828 & 2.807 & 2.593 \\
\hline Tr-Ys & $1 \mathrm{RR}$ & 2.825 & 2.234 & 2.610 & 2.507 \\
\hline Tr-Ys & $2 R R$ & 2.792 & 2.884 & 2.928 & 3.074 \\
\hline Tr-Ys & 3RR & 2.906 & 2.723 & 2.841 & 2.769 \\
\hline Wb-Mc & ORR & 4.510 & 4.436 & 4.000 & 4.378 \\
\hline Wb-Mc & $1 \mathrm{RR}$ & 4.719 & 4.486 & 4.144 & 3.929 \\
\hline Wb-Mc & $2 \mathrm{RR}$ & 4.443 & 4.986 & 3.912 & 4.195 \\
\hline Wb-Mc & 3RR & 5.203 & 5.022 & 4.572 & 4.912 \\
\hline Wb-Ys & ORR & 4.829 & 4.780 & 4.749 & 4.622 \\
\hline Wb-Ys & $1 R R$ & 4.403 & 4.608 & 4.781 & 4.454 \\
\hline Wb-Ys & $2 \mathrm{RR}$ & 4.238 & 3.991 & 4.581 & 4.612 \\
\hline Wb-Ys & 3RR & 4.238 & 3.982 & 4.543 & 4.742 \\
\hline Mc-Ys & ORR & 2.400 & 2.424 & 2.828 & 2.355 \\
\hline Mc-Ys & $1 \mathrm{RR}$ & 2.234 & 2.286 & 2.640 & 2.384 \\
\hline Mc-Ys & 2RR & 2.621 & 2.632 & 2.635 & 2.426 \\
\hline Mc-Ys & $3 R R$ & 2.726 & 2.603 & 2.862 & 2.681 \\
\hline
\end{tabular}

Notes. Station codes: Mc - Medicina, Tr - Toruń, Wb - Westerbork, Ys - Yebes. cavity made of $100 \mathrm{~mm}$-long ultra-low expansion glass spacer and fused silica mirrors. The instability of our clock laser, when it is not referenced to the atomic transition, is below $2 \times 10^{-14}$ on time scales of 2 to $10^{4} \mathrm{~s}$. The clock laser frequency is digitally locked to the frequency of strontium $698 \mathrm{~nm}$ clock transition. The clock laser frequency stabilization is performed by a frequency shifter that also removes slow linear frequency drift of the ultra-stable cavity. The frequency corrections are derived from the excitation probability on both sides of the atomic line (Morzyński et al. 2013). The sample of atoms in the optical lattice is produced and interrogated every $1.3 \mathrm{~s}$, therefore the servo loop cycle in our set-up is equal to $2.6 \mathrm{~s}$. The instability in terms of the Allan deviation of the locked clock laser starts to decrease after few seconds averaging with a slope close to $\tau^{-1 / 2}$, where $\tau$ is the averaging period.

With the frequency comb repetition rate actively stabilized to the optical atomic clock, a $10 \mathrm{MHz}$ signal can be produced by the Direct Digital Synthesizer (DDS) that is inside the comb stabilization loop (see Fig. 7). Hence the $10 \mathrm{MHz}$ signal is locked to the frequency of strontium $698 \mathrm{~nm}$ clock transition and its stability is not deteriorated by any other external RF sources. The $10 \mathrm{MHz}$ signal was subsequently transmitted to the Torun VLBI station over a stabilized fibre link.

We conducted a proof-of-concept experiment on 15 March 2016 during the test time preceding a regular e-VLBI session of the European VLBI Network (EVN). Besides the Torun VLBI station, the participating telescopes included Effelsberg (DE), Medicina (IT), and Yebes (ES). The observing format comprised eight dual-polarization $16 \mathrm{MHz}$ sub-bands, together spanning $4926.49 \mathrm{MHz}$ to $5054.49 \mathrm{MHz}$. The target throughout the observation was the bright radio quasar $0234+285$. The tactics of this test observation were essentially the same as those for the test described in Sect. 3. The two methods for T\&F control at Torun VLBI station were 


\section{Phase for Remote Maser}

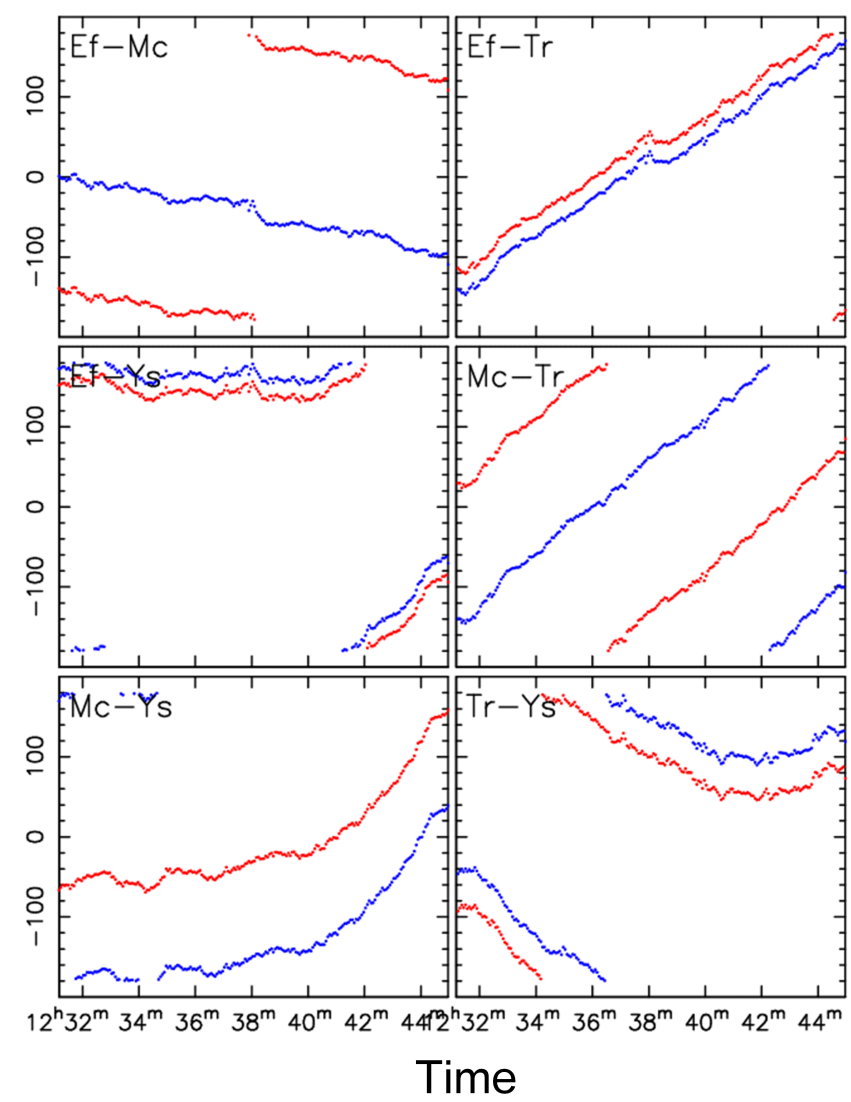

Phase for Optical Clock

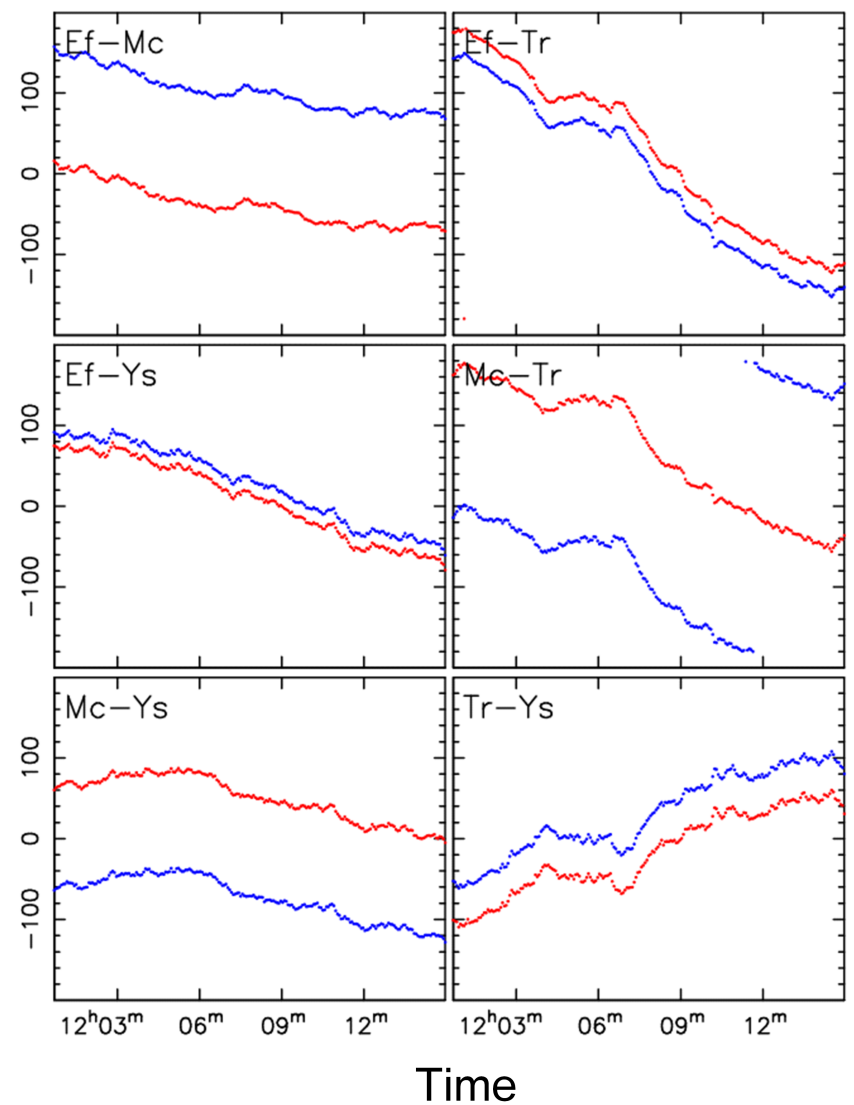

Fig. 8. Residual interferometer phase for two methods of providing T\&F control to the Torun VLBI station during the experiment of 15 March 2016. The $x$-axis is UTC and the $y$-axis is degrees of phase in both polarizations of the fourth sub-band (polarization colour-coding: red $=\mathrm{RCP}$, blue $=$ LCP), vector averaged over the middle $80 \%$ of the sub-band. Each four-second integration interval is plotted as a separate point. The baselines are arranged such that those including Torun are in the right-hand column for each of the T\&F control methods.

the operational remote $\mathrm{HM}$ at $\mathrm{AOS}$ and the optical lattice clock described above. Once the clock-model coefficients were adjusted, we swapped $\mathrm{T} \& \mathrm{~F}$ control to the optical lattice clock for three $15 \mathrm{~min}$ scans and then swapped back to the remote HM. As in the previous test, in addition to the real-time e-VLBI correlation, the raw data from the telescopes were also recorded at JIVE (two 15 min scans for the remote HM).

Figure 8 shows representative behaviour of the residual interferometer phases, plotting one $15 \mathrm{~min}$ scan per domain for both hands of polarization for one sub-band. A feature that is typical of phase variations induced by propagation can be seen around 12:05-12:07 UTC for baselines to Torun in the optical clock plots. At an observing frequency of $5 \mathrm{GHz}$ a cycle of phase corresponds to $200 \mathrm{ps}$, thus the approximately 40 degree hiccup is equivalent to about $22 \mathrm{ps}$, or to about $7 \mathrm{~mm}$ of differential tropospheric path length. We considered the same phasedifference statistics to characterize the phase noise achieved for each method of T\&F control at Torun. Table 2 shows the results for four of the 16 sub-band/polarizations for the case of the difference-pair statistic. Again, we have successfully proved that on these timescales the remote optical atomic clock can provide the operational synchronization of radio telescopes during VLBI observations.

\section{Summary}

We describe a fully operational solution of remote synchronization of the Torun VLBI station with T\&F signals generated in a dedicated UTC laboratory and provided via a stabilized fibre link. We have demonstrated that the remote synchronization does not deteriorate the noise characteristics of the observations and eliminates the issues related to local HM frequency offset and/or drift. In the particular situation of the Torun VLBI station, the obvious benefit is the replacement of the local HM beginning to show signs of imminent failure. This is why the Torun VLBI station has used the remote T\&F delivery in all VLBI observations in which it has participated since the beginning of 2016.

We also show the results of a proof-of-concept experiment with synchronization based on the strontium optical lattice clock, where the optical frequency was down-converted to the RF domain by an optical frequency comb and then delivered to the Torun VLBI station via a stabilized fibre link. This experiment demonstrates the operational usefulness of the optical clock in VLBI observations and may be regarded as a first step towards more complex experiments that might show the noticeable benefits from using optical clocks instead of HMs, the traditional $\mathrm{T} \& \mathrm{~F}$ standards used in VLBI for decades. Our long-term strategy is to deliver an optical carrier instead of $10 \mathrm{MHz}$ signal to the Torun VLBI station and to operate the optical frequency comb and down-conversion system locally at the station. In such circumstances, it would be possible to increase the resulting electrical reference frequency from $10 \mathrm{MHz}$ used in the proofof-concept experiment to much higher value (of the order of several gigahertz). As an ultimate step, we plan to arrange the experimental session in cooperation with at least one other VLBI 
Table 2. Phase noise in picoseconds for different baselines and subbands for remote HM and optical clock.

\begin{tabular}{lccccc}
\hline \hline \multirow{2}{*}{ Baseline } & \multirow{2}{*}{ Sub- } & \multicolumn{2}{c}{ Remote HM } & \multicolumn{2}{c}{ Opt clock } \\
& band & noise & noise & noise & noise \\
& and & rms & rms & rms & rms \\
& polarity & odd & even & odd & even \\
\hline Ef-Tr & 0RR & 1.475 & 1.530 & 1.342 & 1.373 \\
Ef-Tr & 1RR & 1.424 & 1.539 & 1.357 & 1.363 \\
Ef-Tr & 2RR & 1.443 & 1.547 & 1.361 & 1.367 \\
Ef-Tr & 3RR & 1.439 & 1.531 & 1.352 & 1.325 \\
\hline Mc-Tr & 0RR & 1.333 & 1.780 & 1.594 & 1.616 \\
Mc-Tr & 1RR & 1.249 & 1.734 & 1.671 & 1.582 \\
Mc-Tr & 2RR & 1.301 & 1.680 & 1.619 & 1.533 \\
Mc-Tr & 3RR & 1.267 & 1.714 & 1.483 & 1.615 \\
\hline Tr-Ys & 0RR & 2.018 & 2.041 & 1.938 & 1.906 \\
Tr-Ys & 1RR & 1.993 & 1.984 & 1.995 & 1.880 \\
Tr-Ys & 2RR & 1.952 & 2.054 & 2.077 & 1.894 \\
Tr-Ys & 3RR & 2.023 & 2.061 & 2.026 & 1.844 \\
\hline Ef-Mc & 0RR & 1.243 & 1.562 & 0.969 & 1.073 \\
Ef-Mc & 1RR & 1.212 & 1.513 & 0.965 & 1.085 \\
Ef-Mc & 2RR & 1.204 & 1.576 & 0.977 & 1.056 \\
Ef-Mc & 3RR & 1.198 & 1.552 & 1.003 & 1.042 \\
\hline Ef-Ys & 0RR & 1.736 & 1.969 & 1.343 & 1.401 \\
Ef-Ys & 1RR & 1.695 & 1.975 & 1.358 & 1.403 \\
Ef-Ys & 2RR & 1.713 & 1.959 & 1.365 & 1.418 \\
Ef-Ys & 3RR & 1.722 & 1.938 & 1.330 & 1.393 \\
\hline Mc-Ys & 0RR & 1.568 & 1.589 & 1.250 & 1.172 \\
Mc-Ys & 1RR & 1.587 & 1.598 & 1.341 & 1.226 \\
Mc-Ys & 2RR & 1.594 & 1.512 & 1.221 & 1.253 \\
Mc-Ys & 3RR & 1.552 & 1.599 & 1.296 & 1.193 \\
\hline & & & & & \\
& & & & \\
& & &
\end{tabular}

Notes. Station codes: Ef - Effelsberg, Mc - Medicina, Tr - Toruń, Ys - Yebes.

station also synchronized with an optical clock and to perform observation in the millimetre-wave range. These efforts combined would give final evidence that VLBI observations synchronized by optical clocks can outperform those carried out using HMs in terms of stability and accuracy.

We believe that the work presented here, together with other similar experiments described by Clivati et al. (2015), Perini et al. (2016), and Clivati et al. (2017), will start a discussion within the VLBI community on the potential benefits of a traceable, remote synchronization of VLBI stations either using HMs or even more stable and accurate optical clocks.
Acknowledgements. We thank the teams of the Westerbork, Medicina, and Yebes VLBI stations for their willingness to participate in the test of 18 December 2015 organized in the form of a dedicated VLBI session outside the regular EVN time. This work was supported by Polish National Science Center (NCN) under projects Nos. 2014/15/B/ST7/00471 and 2015/17/B/ST7/03628. P. Morzyński was partly supported by Polish National Science Center (NCN) under project No. 2014/15/D/ST2/05281. Support has been received from the project EMPIR 15SIB03 OC18. This project has received funding from the EMPIR programme co-financed by the Participating States and from the European Union's Horizon 2020 research and innovation programme. This research is a part of the programme of the National Laboratory FAMO in Torun, Poland.

\section{References}

Bloom, B. J., Nicholson, T. L., Williams, J. R., et al. 2014, Nature, 506, 71 Bober, M., Morzyński, P., Cygan, A., et al. 2015, Meas. Sci. Technol., 26, 075201 Buczek, L., Kołodziej, J., Krehlik, P., et al. 2015, in Proc. 2015 Joint Conf. IEEE Int. Frequency Control Symp. \& the European Frequency and Time Forum, 583

Calonico, D., Bertacco, E. K., Calosso, C. E., et al. 2014, Appl. Phys. B: Lasers and Optics, 117, 979

Clivati, C., Costanzo, G. A., Frittelli, M., et al. 2015, IEEE Trans. Ultrason. Ferroelectr. Freq. Control, 62, 1907

Clivati, C., Ambrosini, R., Artz, T., et al. 2017, Sci. Rep., 7, 40992

Dierikx, E., Wallin, A. E., Fordell, T., et al. 2016, IEEE Trans. Ultrason. Ferroelectr. Freq. Control, 63, 945

Droste, S., Ozimek, F., Udem, T., et al. 2013, Phys. Rev. Lett., 111, 110801

Falke, S., Lemke, N., Grebing, C., et al. 2014, New J. Phys., 16, 073023

Gordon, D., MacMillan, D., \& Baver, K. 2006, in IVS 2006 General Meeting Proc., eds. D. Behrend, \& K. D. Baver, 291

Hinkley, N., Sherman, J. A., Phillips, N. B., et al. 2013, Science, 341, 1215

Krehlik, P., Śliwczyński, Ł., Buczek, Ł., \& Lipiński, M. 2012, IEEE Trans. Instrum. Meas., 61, 2844

Le Targat, R., Lorini, L., Le Coq, Y., et al. 2013, Nature Comm., 4, 2109

Lopez, O., Amy-Klein, A., Lours, M., Chardonnet, C., \& Santarelli, G. 2010, Appl. Phys. B: Lasers and Optics, 98, 723

Lopez, O., Haboucha, A., Chanteau, B., et al. 2012, Opt. Express, 20, 23518

Ma, L. S., Jungner, P. A., Ye, J., \& Hall, J. L. 1994, Opt. Lett., 19, 1777

Morzyński, P., Wcisło, P., Ablewski, P., et al. 2013, Opt. Lett., 38, 4581

Morzyński, P., Bober, M., Bartoszek-Bober, D., et al. 2015, Sci. Rep., 5, 17495

Newbury, N. R., \& Swann, W. C. 2007, J. Opt. Soc. Am. B, 24, 1756

Perini, F., Bortolotti, C., Roma, M., et al. 2016, in IVS 2016 General Meeting Proc., eds. D. Behrend, K. D. Baver, \& K. L. Armstrong, 166

Rioja, M., Dodson, R., Asaki, Y., Hartnett, J., \& Tingay, S. 2012, AJ, 144, 121

Rogers, A. E. E., \& Moran, J. M. 1981, IEEE Trans. Instrum. Meas., IM-30, 283

Śliwczyński, Ł., Krehlik, P., \& Lipiński, M. 2010, Meas. Sci. Technol., 21, 075302

Śliwczyński, Ł., Krehlik, P., Buczek, Ł., \& Lipiński, M. 2011, IEEE Trans. Instrum. Meas., 60, 1480

Śliwczyński, Ł., Krehlik, P., Czubla, A., Buczek, Ł., \& Lipiński, M. 2013, Metrologia, 50, 133

Śliwczyński, Ł., Krehlik, P., Lipiński, M., Turza, K., \& Binczewski, A. 2015, Proc. Joint IEEE Int. Frequency Control Symp. \& the European Frequency and Time Forum, 280

Śliwczyński, Ł., Krehlik, P., Turza, K., \& Binczewski, A. 2016, in Proc. 30th European Frequency and Time Forum, 1009

Thompson, A. R., Moran, J. M., \& Swenson, G. W. 1986, Interferometry and Synthesis in Radio Astronomy (New York: John Wiley and Sons)

Ushijima, I., Takamoto, M., Das, M., Ohkubo, T., \& Katori, H. 2015, Nature Photonics, 9, 185 